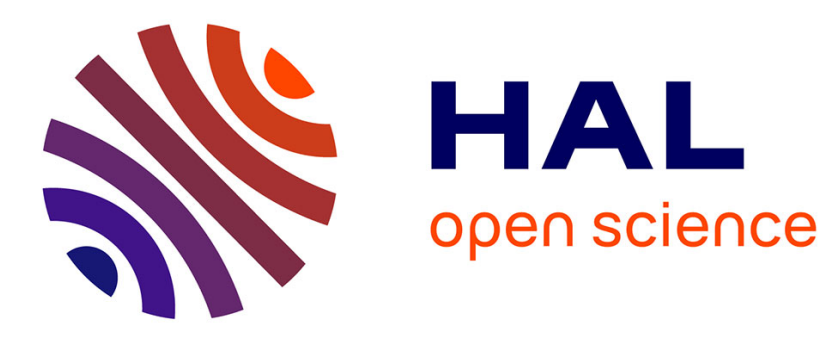

\title{
Évolution sur 12 ans des indications de prostatectomie totale pour cancer
}

J. Rose Dite Modestine, Y. Neuzillet, J.-M. Herve, P.-O. Bosset, A. Abdou, D. Bohin, T. Ghoneim, M. Rouanne, C. Radulescu, T. Lebret

\section{To cite this version:}

J. Rose Dite Modestine, Y. Neuzillet, J.-M. Herve, P.-O. Bosset, A. Abdou, et al.. Évolution sur 12 ans des indications de prostatectomie totale pour cancer. Progrès en Urologie, 2019, 29, pp.408 - 415. 10.1016/j.purol.2019.06.006 . hal-03487970

\section{HAL Id: hal-03487970 \\ https://hal.science/hal-03487970}

Submitted on 20 Dec 2021

HAL is a multi-disciplinary open access archive for the deposit and dissemination of scientific research documents, whether they are published or not. The documents may come from teaching and research institutions in France or abroad, or from public or private research centers.
L'archive ouverte pluridisciplinaire HAL, est destinée au dépôt et à la diffusion de documents scientifiques de niveau recherche, publiés ou non, émanant des établissements d'enseignement et de recherche français ou étrangers, des laboratoires publics ou privés.

\section{(ㄷ)(1) $\$$}

Distributed under a Creative Commons Attribution - NonCommercial| 4.0 International 


\section{Évolution sur 12 ans des indications de chirurgie radicale pour le traitement du cancer de la prostate.}

\section{2-year history of radical surgery indications for the treatment of prostate cancer.}

Johan ROSE-DITE-MODESTINE ${ }^{1}$, Yann NEUZILLET ${ }^{1}$, Jean-Marie HERVE ${ }^{1}$, Pierre-Olivier BOSSET $^{1}$, Abdelali ABDOU ${ }^{1}$, Denis BOHIN ${ }^{1}$, Tarek GHONEIM ${ }^{1}$, Mathieu ROUANNE ${ }^{1}$, Camélia RADULESCU ${ }^{2}$, Thierry LEBRET $^{1}$

1. Université de Versailles - Saint-Quentin-en-Yvelines, hôpital Foch, service d'urologie et de transplantation rénale, 40 rue Worth, 92150 Suresnes, France.

2. Hôpital Foch, service d'anatomopathologie, 40 rue Worth, 92150 Suresnes, France.

Auteur correspondant : $\quad$ Dr. Johan ROSE-DITE-MODESTINE, MD

Service d'Urologie et Transplantation Rénale

Université de Versailles - Saint-Quentin-en-Yvelines

Hôpital Foch

40 , rue Worth

92150 SURESNES

Tél. : 0146252465

Mail : j.rose-dite-modestine@hopital-foch.com 


\section{Introduction :}

L'incidence du cancer de la prostate rapportée en France en 2015 par le réseau Francim a été de 50430 nouveau cas [1]. Parmi les options thérapeutiques du cancer de la prostate localisée, la chirurgie occupe en France une place prépondérante avec un nombre de prostatectomie radicale estimée à 20000 par an par l'Institut de recherche et documentation en économie de la santé. Toutefois, les données actualisées du PMSI montre que depuis 10 ans, le nombre de prostatectomie radicale réalisées en France a diminué de $35 \%$ et se stabilise. Conformément aux directives de la Haute Autorité de Santé, les décisions de traitement du cancer de la prostate sont discutées de façon collégiale au cours de réunion de concertation pluridisciplinaire (RCP). Avant d'être soumise, expliquée au patient et acceptée, la proposition de prostatectomie radicale et de curage ganglionnaire qui peut lui être associée est donc confrontée au référentiel de la RCP qui s'appuie en France sur les recommandations du Comité de Cancérologie de l'Association Française d'Urologie (CCAFU). Ces recommandations font l'objet de révision régulières par les sociétés savantes qui les émettent et modifient les indications du traitement. Ainsi, en 2007, les recommandations du CCAFU indiquaient la prostatectomie radicale comme " un traitement efficace d'un cancer de la prostate de stades (T1a), T1b, T1c et $T 2$ » [2]. Pour les stades plus avancés, le CCAFU publiait que : « La prostatectomie isolée peut être indiquée pour les stades T3 limités. Pour les stades T3 étendus et pN1, elle est associée à un taux de récidive biologique à 5 ans supérieur à 70\% ». Quant à la place du curage ganglionnaire, le CCAFU l'indiquait quand le risque élevé (> $5 \%$ ) de métastases ganglionnaires était défini par un PSA $>10 \mathrm{ng} / \mathrm{ml}$, et/ou un score de Gleason $>7$ ( $4+3$ ou un \% de grade $4>50 \%$ ), et/ou une imagerie des aires ganglionnaires suspecte [3]. Les recommandations émissent en 2010, évoluèrent modérément, statuant que: «classiquement réservée aux tumeurs intracapsulaires de risque faible ou intermédiaire, la prostatectomie totale peut également s'envisager dans certaines conditions pour des tumeurs à risque élevé notamment avec une extension extra-capsulaire limitée (T3a clinique, biopsique ou IRM), en particulier chez des patients jeunes et demandeurs. " [4]. Au sujet du curage, le CCAFU ajoutait alors que " en cas de risque intermédiaire ou élevé, un curage lympho-nodal est recommandé, si le choix d'une prostatectomie totale a été retenu. En cas de risque faible, le curage ganglionnaire peut ne pas être effectué ». Suite à une analyse de la littérature publiée en 2012 [5], l'indication du curage ganglionnaire en cas de faible risque fut amandée dans l'édition 2013 
des recommandations du CCAFU, devenant optionnelle [6]. En 2016, les recommandations concernant l'indication de la prostatectomie radicale furent sensiblement modifiées, le CCAFU publiant que: "ses indications sont celle du traitement curatif d'un cancer de prostate localisé ou localement avancé. La prostatectomie radicale est envisageable dans les tumeurs de risque faible, elle est indiquée dans les tumeurs de risque intermédiaire et peut être proposée dans les tumeurs de haut risque avec la possibilité d'un traitement multimodal associé » [7]. Les indications du curage ganglionnaires ont été également revues, le CCAFU statuant que le curage ganglionnaire n'est pas indiqué dans le groupe faible risque, doit être réalisé dans le groupe intermédiaire en cas de risque $>5 \%$ et doit être réalisé dans le groupe haut risque. L'actualisation des recommandations publiée récemment a conforté ces indications [8].

La présente étude a analysé les indications de prostatectomie radicale et de curage ganglionnaire retenue en RCP au cours des 12 dernières années dans un centre chirurgical hospitalo-universitaire francilien afin d'évaluer leur adéquation vis-à-vis des recommandations du CCAFU concomitamment en vigueur.

\section{Matériel et Méthode :}

Les données démographiques, cliniques et pathologiques de tous les patients traités par prostatectomie radicale entre le 16/03/2007 et le 17/03/2019 dans un centre chirurgical hospitalo-universitaire francilien ont été collectées prospectivement (recueil des données approuvé par le CPP N¹30207). Les indications opératoires de prostatectomie totale et de curage ganglionnaire associé ont été validées en réunion de concertation pluridisciplinaire, soit en accord avec le référentiel (recommandation d'onco-urologie du Comité de Cancérologie de l'Association Française d'Urologie en vigueur), soit par avis collégial après discussion du cas. Les Information recueillies ont été l'âge du patient au moment de l'intervention, ont été l'âge, le poids et la taille des patients et leurs comorbidités (cardiopathie ischémique et diabète). Les données biologiques, anatomopathologiques et clinique définissants les groupes de risque de la classification de D’Amico [9] ont été consignés (valeur du PSA sérique, stade clinique, score de Gleason sur la ponction biopsie prostatique) permettant ainsi le classement de chaque patient selon qu'il soit de risques faible (PSA $<10 \mathrm{ng} / \mathrm{ml}$ et score de Gleason $6(3+3)$ et stade $\mathrm{cT} \leq 2 \mathrm{a}$ ), intermédiaire (PSA entre 
10 et $20 \mathrm{ng} / \mathrm{ml}$, score de Gleason 7, stade cT2b) ou haut (PSA > $20 \mathrm{ng} / \mathrm{ml}$ ou score de Gleason $\geq 8(4+4)$ ou stade $c T \geq 2 c)$.

Les patients ont été opérés par des opérateurs urologues séniors $(n=8)$ ou par des urologues chefs de clinique ( $n=2)$ avec l'assistance d'un urologue sénior.

Les pièces de prostatectomies et de curage ganglionnaire ont été analysées ou revues par une seule uro-anatomopathologiste. Le stade pathologique, le statut des marges chirurgicales, le score de Gleason et le poids de la pièce opératoire ont été consignés.

L'analyse rétrospective de la base de données constituée a été effectuée en mai 2019 après récupération des données de l'analyse anatomopathologiques des pièces opératoires. Les variables quantitatives ont été exprimées par leur moyenne et leur écart-type (DS). Les variables qualitatives ont été décrites par leur fréquence en pourcentage. Les patients ont été répartis en quatre groupes selon la période de 3 ans où ils ont été traités, distinguant ainsi ceux opérés entre 2007 et 2010, entre 2010 et 2013, entre 2013 et 2016 et entre 2016 et 2019. L'adéquation de la réalisation ou non d'un curage ganglionnaire en fonction des recommandations en vigueur a été analysée par mise en rapport de la réalisation d'un curage à l'indication de ce curage selon les recommandations. Les variables quantitatives étaient comparées par le test $\mathrm{t}$ de Student. Les variables qualitatives étaient comparées par le test $\mathrm{du} \mathrm{Chi}^{2}$. Tous les tests statistiques étaient bilatéraux, les variances des groupes comparés étaient considérées comme égales, le seuil de significativité était fixé à $5 \%$. La tendance monotonique sur les 4 périodes a été évaluée par application d'un test de MannKendall. Les analyses statistiques étaient réalisées à l'aide du logiciel Microsoft Excel et de XLSTAT. 


\section{Résultats :}

Deux mille quatre-vingt-huit patients consécutifs traités par prostatectomie radicale entre le 16/03/2007 et le 17/03/2019 ont été inclus. Le nombre de patient inclus par période de 3 ans a été de 279 entre 2007 et 2010, 605 entre 2010 et 2013, 688 entre 2013 et 2016 et 716 entre 2016 et 2019. Les caractéristiques cliniques et pathologiques de la population de l'étude ont été rapporté pour son intégralité et pour chaque période de 3 ans considérées dans le tableau 1.

L'analyse comparative des données démographiques par période a montré une différence d'âge des patients inclus pendant la période 2007-2010, plus jeunes que ceux des 3 autres périodes. Les patients de la période 2007-2010 étaient également moins fréquemment porteurs d'une cardiopathie ischémique ou d'un diabète.

La proportion de patients classés à risque faible, intermédiaire ou haut selon D'Amico a été de $13,2 \%, 80,8 \%$ et $6,0 \%$ respectivement. L'analyse comparative par période a montré une fréquence décroissante de patient de faible risque, passant de $16,8 \%$ à $11,3 \%$, avec une fréquence significativement moindre sur les périodes 2013-2016 et 2016-2019 comparativement aux deux précédentes [Figure 1]. Inversement, la fréquence des patients classés à haut risque selon D'Amico a augmenté de 3,9\% à 9,6\% et a été supérieure pendant la période 2016-2019 par rapport aux trois précédentes.

L'analyse comparative par périodes des 3 critères définissants les groupes de la classification de D’Amico a montré que la distribution des scores de Gleason des patients opérés a varié au cours du temps. Une réduction de la fréquence des scores de Gleason $6(3+3)$ a été observée, passant de $52,0 \%$ à $17,8 \%$. Inversement, la fréquence des scores de Gleason $7(4+3)$ et $\geq 8(4+4)$ a augmenté, passant respectivement de $10,8 \%$ à $20,8 \%$ et de $4,7 \%$ à $7,0 \%$. Concernant le stade clinique, une réduction de la fréquence des patients dont le stade était cT $\leq 2 \mathrm{a}$ a été observée, passant de $76,0 \%$ à $68,2 \%$, avec fréquence significativement moindre sur la période 2016-2019 comparativement aux trois précédentes. Subséquemment, l'analyse des stades pathologiques et du statut des marges chirurgicales déterminés sur les pièces opératoires ont montré une fréquence croissante de stade avancée et d'atteinte des marges. Les différences observées concernant les valeurs de PSA ont été uniquement une fréquence moindre de patient ayant un PSA > $20 \mathrm{ng} / \mathrm{ml}$ pendant la période 2007-2010 comparativement aux périodes ultérieures. Toutefois, au cours des 12 années étudiées, plus de $95 \%$ des patients opérés avaient une valeur de PSA $<20 \mathrm{ng} / \mathrm{ml}$. 
Un curage ganglionnaire a été associé à la prostatectomie radicale pour $14,0 \%$ des patients à faible risque, $71,6 \%$ des patients de risque intermédiaire et $96,3 \%$ des patients à haut risque et a montré un envahissement ganglionnaire dans respectivement $0,0 \%, 7,0 \%$ et $25,5 \%$ des cas. Ces fréquences n'ont pas varié au cours du temps. L'analyse de l'adéquation de la réalisation ou non d'un curage ganglionnaire en fonction des recommandations en vigueur a montré que l'indication était adéquate dans $96,3 \%$ des cas pour les patients à haut risque, $83,3 \%$ des cas pour les patients à faible risque et $41,8 \%$ pour les patients de risque intermédiaire. Ces fréquences n'ont pas varié au cours du temps.

\section{Discussion :}

Au cours des 12 dernières années, l'évolution des connaissances médicales ont conduit à une migration des indications de la prostatectomie radicale et du curage ganglionnaire qui peut lui être associé.

Les patients à faible risque selon la classification de D'Amico peuvent répondre aux critères d'éligibilité pour une surveillance active, un traitement par curiethérapie interstitielle exclusive ou, dans le cadre d'une étude clinique observationnelle (étude HIFI), au traitement par ultrasons focalisés de haute intensité (HIFU). Ces alternatives thérapeutiques, dont les patients attendent une moindre morbidité [10] sont compétitives et réduisent le nombre de traitement par prostatectomies radicale. Notre étude a montré une réduction progressive et significative du nombre de patients opéré pour un cancer de la prostate de faible risque, passant de $16,8 \%$ à $11,3 \%$. Le détail de l'analyse des critères définissant les groupes de risque de la classification de D'Amico a montré que le score de Gleason a été un élément majeur dans la décision prise en RCP. La proportion de patient opéré pour un cancer de score de Gleason $6(3+3)$ a diminué de $66 \%$, passant $52,0 \%$ sur la période $2007-2010$ à $17,8 \%$ sur la période 2016-2019. Parallèlement, la proportion des patients présentant une tumeur de stade cT $\geq 2 \mathrm{a}$ a diminué de $7 \%$ et celle des patients ayant un PSA $<10 \mathrm{ng} / \mathrm{ml}$ n'a pas varié. Chez ces patients à faible risque, la part de ceux qui ont été traité par un curage ganglionnaire en association à la prostatectomie a été de $14 \%$ et n'a pas varié avec les différentes actualisations des recommandations du CCAFU. Les indications de curage chez ces patients ont ainsi été conformes aux recommandations, c'est-à-dire consistant en son éviction, dans $83,3 \%$ des cas. L'analyse des pièces opératoires rapportée par notre étude plaide en faveur d'une meilleure sélection des cancers opérés selon les dernières 
recommandations, avec une fréquence de cancer de score de Gleason 6(3+3) en diminution de $87 \%$, passant de $26,9 \%$ en 2007-2010 à 3,5\% en 2016-2019. L'absence de dissémination ganglionnaire mise en évidence chez les patients à faible risque conforte l'attitude recommandée de surseoir à la réalisation du curage ganglionnaire.

Pour les patients ayant un cancer à haut risque selon la classification de D'Amico, l'indication de prostatectomie radicale associée à un curage ganglionnaire a été introduite dans les recommandations CCAFU en 2010 et confortée dans les actualisations ultérieures avec la possibilité d'un traitement multimodal associé. Pour ces patients, l'alternative recommandée dans un but curatif est le traitement par irradiation externe associé à une hormonothérapie. Les modalités de ce traitement par radio-hormonothérapie ont évolué durant les 12 années sur lesquelles porte notre étude, avec principalement une réduction de la durée de l'hormonothérapie à 18 mois au lieu de 36 mois [11]. Cette réduction de la suppression androgénique aurait pu favoriser son choix en RCP et augmenter l'adhésion des patients. Cela n'a pas été le cas dans notre étude, où nous avons observé une augmentation progressive et significative du nombre de patients opérés pour un cancer de la prostate de haut risque, passant de $3,9 \%$ à $9,6 \%$. Le détail de l'analyse des critères définissant les groupes de risque de la classification de D'Amico a montré que les indications supplémentaires de prostatectomie étaient principalement retenues pour des patients ayant un cancer de score de Gleason plus élevé. La proportion de patient opéré pour un cancer de score de Gleason $\geq 8(4+4)$ a augmenté de $49 \%$, passant 4,7\% sur la période 2007-2010 à 7,0\% sur la période 2016-2019. Parallèlement, la proportion des patients présentant une tumeur de stade $c T \geq 2 \mathrm{c}$ a augmenté de $20 \%$ et celles des patients ayant un PSA $>20 \mathrm{ng} / \mathrm{ml}$ n'a varié qu'à la marge (3,2\% en 2007-2010 vs. 5,1\% en 2013-2016, décroissant ensuite). Chez ces patients à haut risque, notre étude a rapporté une adéquation de la pratique du curage ganglionnaire conformément aux recommandations de plus de $95 \%$. La fréquence de l'envahissement ganglionnaire sur l'analyse du curage, rapporté à $25,5 \%$ dans notre étude, conforte cette attitude.

L'étude de l'évolution des indications de chirurgie radicale pour le traitement du cancer de la prostate a fait l'objet d'une étude entre 1989-1990 et 2001-2002 au sein du registre nordaméricain CaPSURE, montrant une migration des indications en faveur des tumeurs de plus faible risque [12]. L'étude de Gallina et coll. colligeant 11350 cas de patients traité par 
prostatectomie radicale entre 1988 et 2005 dans 3 centres européens et 3 centres nordaméricains a confirmé cette migration de stade des cancers opérés en Europe [13]. Les auteurs rapportaient une augmentation de $67 \%$ des patients opérés pour des cancers de stade cT1c. Ces séries anciennes ne correspondent plus aux situations contemporaines de diagnostic du cancer de la prostate. Plus récemment, Mitsuzuka et coll. ont rapporté une évolution similaire dans une population japonaise de 1268 patients opérés dans 4 centres entre 2000 et 2009 [14]. Les auteurs nippons ont rapporté une diminution en fréquence des cancers de haut risque de D'Amico, passant de $42 \%$ à $18 \%$. Deux analyses du registre nordaméricain SEER ont également été récemment rapportées. La première, portant sur 24790 patients opérés entre 2000 et 2015 a montré, comme notre étude, une diminution de la proportion de prostatectomies radicales faite en situation où une surveillance active était proposable passant de $31 \%$ à $5 \%$ [15]. Inversement, la proportion d'intervention réalisées pour des cancers classés à haut risque a augmenté, passant de $10 \%$ à $30 \%$. La seconde, portant sur 58558 patients opérés plus récemment, entre 2010 et 2014, a montré une augmentation significative de la proportion de prostatectomies radicales réalisées pour des cancer de la prostate non localisé, passant de $18,7 \%$ à $24,2 \%$ [16]. La proportion de patient présentant un score de Gleason $\geq 7(4+3)$ a également été observé à la hausse, passant de 10,8\% à 14,2\%. Concernant la population française, une étude bi-centrique a été rapportée par Patard PM et coll., colligeant les prostatectomies réalisée en 2005, 2010 et 2015 et comparant la distribution des patients en fonction des groupes de la classification de D’Amico [17]. Les résultats portant sur un total de 1282 patients sont concordant avec ceux de notre étude. Notre série, monocentrique mais cumulant un nombre supérieur de patients sur une durée d'étude continue rapporte donc des résultats originaux et correspondant à ceux attendu eu égard à l'évolution des recommandations de bonnes pratiques clinique. Notre étude est cependant la première à contextualiser les migrations d'indications de prostatectomies radicales vis-à-vis des recommandations en vigueur au moment du choix thérapeutique.

Les migrations d'indications de prostatectomies radicales, en accord avec celles retenue par les recommandations du CCAFU, sont double. Concernant les patients à haut risque, elles reposent principalement sur un rationnel d'une diminution du risque de complication liée à l'évolution locale du cancer de la prostate. Secondairement, elles peuvent être soutenues 
par l'hypothèse d'une réduction du risque de dissémination métastatique du cancer de la prostate associé à la cytoréduction chirurgicale [18]. Concernant les patients à faible risque, elles sont fondées sur les résultats de la surveillance active qui assure une sécurité oncologique équivalente à celle de la prostatectomie radicale chez ces patients [19].

Notre étude présente les limites inhérentes à son caractère rétrospectif et monocentrique. Le recueil prospectif des données et le contrôle de qualité du recueil reposant sur un seul investigateur tout au long des 12 années limite le risque de biais. Le volume d'activité chirurgical du centre hospitalo-universitaire où a été mené notre étude, a été assuré par 8 urologues sénior et 2 chefs de clinique pour les prostatectomies radicales. La pluralité des avis lors des discussions autour du référentiel que constituait les recommandations CCAFU en vigueur compense partiellement le caractère monocentrique de l'étude. Toutefois, une étude de méthodologie comparable portant sur un nombre plus important de centre serait nécessaire pour confirmer les conclusions de la présente étude.

\section{Conclusion :}

Les indications de prostatectomies radicales et de curages ganglionnaires retenues en RCP au cours des 12 dernières années dans un centre chirurgical hospitalo-universitaire francilien ont évolué. Une part plus importante a été progressivement donné aux traitements des cancers à haut risque selon D'Amico. Chez ces patients à haut risque, le curage ganglionnaire a été associé à la prostatectomie radicale dans plus de $95 \%$ des cas. Parallèlement, la proportion de cancer de faible risque traités par prostatectomie radicale a diminué. Chez ces patients à faible risque, le curage ganglionnaire a été réalisé dans $14 \%$ des cas sans montrer d'envahissement ganglionnaire. Cette étude démontre ainsi une adéquation des indications du traitement chirurgical radical du cancer de la prostate vis-à-vis des recommandations du CCAFU concomitamment en vigueur. 


\section{Références :}

1. Grosclaude P, Remontet L, Daubisse Marliac L, Velten M, Uhry Z, Leone N. Le cancer de la prostate : incidence, survie et mortalité en France. Numéro thématique. Cancer de la prostate : actualité épidémiologique en France. Bull Epidemiol Hebd. 2016;3940:693-9.

2. Soulie M, Beuzeboc P, Richaud P, Villers A, Kassab-Chahmi D, Bataillard A; Association Francaise d'Urologie; Fédération Nationale des Centres de Lutte contre le Cancer. Bulletin de synthèse de veille 2005 Recommandations pour la pratique clinique. Prise en charge thérapeutique du cancer de la prostate non métastatique. Prog Urol. 2007;17:801-9.

3. Peneau M, Villers A, Molinié V, Theis D, Soulié M. Indications de la lymphadénectomie ilio-obturatrice dans le cancer de la prostate cliniquement localisé. Prog Urol. 2004 ;14:287-94.

4. Salomon L, Azria D, Bastide $C$, Beuzeboc $P$, Cormier L, Cornud F, Eiss D, Eschwège $P$, Gaschignard N, Hennequin C, Molinié V, Mongiat Artus $P$, Moreau JL, Péneau M, Peyromaure M, Ravery V, Rebillard X, Richaud P, Rischmann P, Rozet F, Staerman F, Villers A, Soulié M; Oncology Committee of the French Association of Urology (CCAFU). Recommandations en Onco-Urologie 2010 : Cancer de la prostate. Prog Urol. 2010;20:S217-51.

5. Salomon L, Peyromaure M, Fromont G, Rozet F, Eiss D, Bastide C, Beuzeboc $P$, Gachignard N, Cormier L, Hennequin C, Mongiat-Artus P, Souliv $\subset$ M; sous-comitv $\subset$ prostate du CCAFU; Committee of cancerology of the French Association of Urology. Le curage ganglionnaire dans le cancer de la prostate : une mise au point du comité de cancérologie de l'association française d'urologie. Prog Urol. 2012;22:510-9

6. Salomon L, Bastide C, Beuzeboc $P$, Cormier L, Fromont G, Hennequin C, MongiatArtus P, Peyromaure M, Ploussard G, Renard-Penna R, Rozet F, Azria D, Coloby $P$, Molinié V, Ravery V, Rebillard X, Richaud P, Villers A, Soulié M; Les membres du CCAFU. Recommandations en onco-urologie 2013 du CCAFU : Cancer de la prostate. Prog Urol. 2013;23:S69-101

7. Rozet $F$, Hennequin $C$, Beauval JB, Beuzeboc $P$, Cormier L, Fromont $G$, Mongiat-Artus P, Ouzzane A, Ploussard G, Azria D, Brenot-Rossi I, Cancel-Tassin G, Cussenot O, Lebret $T$, Rebillard X, Soulié $M$, Renard-Penna R, Méjean A. Recommandations en onco-urologie 2016-2018 du CCAFU : Cancer de la prostate. Prog Urol. 2016;27:S95S143.

8. Rozet F, Hennequin C, Beauval JB, Beuzeboc P, Cormier L, Fromont-Hankard G, Mongiat-Artus P, Ploussard G, Mathieu R, Brureau L, Ouzzane A, Azria D, Brenot-Rossi I, Cancel-Tassin G, Cussenot O, Rebillard X, Lebret T, Soulié M, Renard Penna R, Méjean A. Recommandations françaises du Comité de Cancérologie de l'AFU pour le cancer de la prostate - actualisation 2018-2020 : cancer de la prostate. Prog Urol. 2018;28:S79-S130.

9. D'Amico AV, Whittington R, Malkowicz SB, Schultz D, Blank K, Broderick GA, Tomaszewski JE, Renshaw AA, Kaplan I, Beard CJ, Wein A. Biocheminical outcome after radical prostatectomy, external beam radiation therapy, or interstitial radiation therapy for clinicaly localized prostate cancer, JAMA 1998;280:969-74 
10. Soulié M, Salomon L. Comparaison des résultats oncologiques et fonctionnels de la chirurgie avec les autres traitements curatifs du cancer de la prostate. Prog Urol. 2015;25:1067-85.

11. Nabid A, Carrier N, Martin AG, Bahary JP, Lemaire C, Vass S, Bahoric B, Archambault R, Vincent F, Bettahar R, Duclos M, Garant MP, Souhami L. Duration of Androgen Deprivation Therapy in High-risk Prostate Cancer: A Randomized Phase III Trial. Eur Urol. 2018 Oct;74(4):432-441.

12. Cooperberg MR, Lubeck DP, Mehta SS, Carroll PR; CaPSURE. Time trends in clinical risk stratification for prostate cancer: implications for outcomes (data from CaPSURE). J Urol. 2003;170:S21-5

13. Gallina A, Chun FK, Suardi N, Eastham JA, Perrotte P, Graefen M, Hutterer G, Huland $H$, Klein EA, Reuther A, Montorsi F, Briganti A, Shariat SF, Roehrborn CG, de la Taille A, Salomon L, Karakiewicz PI. Comparison of stage migration patterns between Europe and the USA: an analysis of 11350 men treated with radical prostatectomy for prostate cancer. BJU Int. 2008;101:1513-8.

14. Mitsuzuka K, Koie T, Narita S, Kaiho Y, Yoneyama T, Tsuchiya N, Kakoi N, Kawamura S, Tochigi T, Ohyama C, Habuchi T, Yamaguchi T, Arai Y. Changes in indications and oncological outcomes of radical prostatectomy after 2000--data from 1268 Japanese patients treated with radical prostatectomy between 2000 and 2009. Jpn J Clin Oncol. 2013;43:821-6.

15. van den Bergh R, Gandaglia G, Tilki D, Borgmann H, Ost P, Surcel C, Valerio M, Sooriakumaran P, Salomon L, Briganti A, Graefen M, van der Poel H, de la Taille A, Montorsi F, Ploussard G; European Association of Urology Young Academic Urologists Working Party on Prostate Cancer (EAU-YAUWP). Trends in Radical Prostatectomy Risk Group Distribution in a European Multicenter Analysis of 28572 Patients: Towards Tailored Treatment. Eur Urol Focus. 2019 Mar;5(2):171-178.

16. Preisser F, Marchioni M, Nazzani S, Bandini M, Tian Z, Saad F, Pompe RS, Briganti A, Budäus L, Montorsi F, Huland H, Graefen M, Tilki D, Karakiewicz PI. Trend of Adverse Stage Migration in Patients Treated with Radical Prostatectomy for Localized Prostate Cancer. Eur Urol Oncol. 2018;1:160-168.

17. Patard PM, Roumiguié $M$, Prudhomme T, Doumerc N, Thoulouzan M, Gamé $X$, de la Taille A, Rischmann P, Soulié M, Salomon L, Beauval JB. Migration in last decade to high-risk prostate cancer after radical prostatectomy. Prog Urol. 2019;29:29-35.

18. Dell'Oglio P, Stabile A, Gandaglia G, Zaffuto E, Fossati N, Bandini M, Suardi N, Karakiewicz PI, Shariat SF, Montorsi F, Briganti A. New surgical approaches for clinically high-risk or metastatic prostate cancer. Expert Rev Anticancer Ther. 2017 ;17:1013-1031.

19. Bill-Axelson A, Holmberg L, Garmo H, Taari K, Busch C, Nordling S, Häggman M, Andersson SO, Andrén O, Steineck G, Adami HO, Johansson JE. Radical Prostatectomy or Watchful Waiting in Prostate Cancer - 29-Year Follow-up. N Engl J Med. 2018;379:2319-2329. 


\section{Légendes :}

Tableau 1 : Caractéristiques cliniques et pathologiques de la population étudiée.

Table 1: Clinical and pathological characteristics of the studied population.

Figure 1 : Proportion de cancer de la prostate opérés selon leur classification par groupe de D’Amico.

Figure 1: Proportion of prostate cancer operated according to their D'Amico group classification. 
Tableau 1 : Caractéristiques cliniques et pathologiques de la population étudiée.

\begin{tabular}{|c|c|c|c|c|c|c|c|}
\hline & $\begin{array}{c}\text { Globale } \\
(n=2288)\end{array}$ & $\begin{array}{c}2007-2010 \\
(n=279)\end{array}$ & $\begin{array}{c}2010-2013 \\
(n=605)\end{array}$ & $\begin{array}{c}2013-2016 \\
(n=688)\end{array}$ & $\begin{array}{c}2016-2019 \\
(n=716)\end{array}$ & $p=$ & $\begin{array}{c}\text { Tendance } \\
(p=)\end{array}$ \\
\hline Âge (années) \pm écart type & $64 \pm 6$ & $62 \pm 6 *$ & $63 \pm 6+$ & $64 \pm 6$ & $64 \pm 6$ & $\begin{array}{l}*=0,01 \\
+<0,001\end{array}$ & \\
\hline Poids $(\mathrm{kg}) \pm$ écart type & $80,5 \pm 12,0$ & $80,8 \pm 12,1$ & $80,2 \pm 12,1$ & $80,8 \pm 12,4$ & $80,4 \pm 11,6$ & & \\
\hline Taille $(m) \pm$ écart type & $1,75 \pm 0,14$ & $1,75 \pm 0,07$ & $1,75 \pm 0,07$ & $1,74 \pm 0,07$ & $1,75 \pm 0,06$ & & \\
\hline IMC $\left(\mathrm{kg} / \mathrm{m}^{2}\right) \pm$ écart type & $26,4 \pm 3,5$ & $26,5 \pm 3,5$ & $26,3 \pm 3,8$ & $26,5 \pm 3,5$ & $26,2 \pm 3,3$ & & \\
\hline $\begin{array}{l}\text { Antécédents (\%): } \\
\text { Cardiopathie ischémique } \\
\text { Diabète }\end{array}$ & $\begin{array}{c}10,3 \\
8,7 \\
\end{array}$ & $\begin{array}{l}8,2 * \\
4,7 \bullet\end{array}$ & $\begin{array}{l}10,5 \\
7,2+\end{array}$ & $\begin{array}{l}11,2 \\
10,6\end{array}$ & $\begin{array}{l}9,9 \\
9,8 \\
\end{array}$ & $\begin{array}{l}*=0,03 \\
\bullet<0,001 \\
+<0,001\end{array}$ & \\
\hline \multicolumn{8}{|l|}{ Biopsies prostatiques } \\
\hline $\begin{array}{l}\text { Score de Gleason }(\%): \\
6(3+3)-\text { ISUP } 1 \\
7(3+4)-\text { ISUP } 2 \\
7(4+3)-\text { ISUP } 3 \\
\geq 8(4+4)-\text { ISUP } \geq 4\end{array}$ & $\begin{array}{c}32,6 \\
43,7 \\
17,3 \\
5,9\end{array}$ & $\begin{array}{c}52,0 * \\
32,6 \\
10,8 \\
4,7\end{array}$ & $\begin{array}{c}49,1+ \\
31,1 \\
13,9 \\
5,5\end{array}$ & $\begin{array}{c}25,6 \\
48,5 \\
19,2^{\bullet} \\
5,8^{\circ}\end{array}$ & $\begin{array}{c}17,8 \\
54,1 \\
20,8^{\#} \\
7,0^{\Omega}\end{array}$ & $\begin{array}{l}*<0,001 \\
\bullet<0,001 \\
+<0,001 \\
\#<0,001 \\
{ }^{\circ}=0,003 \\
\Omega=0,02\end{array}$ & $\begin{array}{l}У(p<0,001) \\
\nearrow(p<0,001) \\
\nearrow(p=0,002) \\
\nearrow(p<0,001)\end{array}$ \\
\hline $\begin{array}{l}\text { Prédominance du grade } 4 \\
\text { (i.e. ISUP } \geq 3 \text { ) (\%) }\end{array}$ & 23,4 & $15,4 *$ & $19,3+$ & 25,4 & 27,9 & $\begin{array}{l}*<0,001 \\
+<0,001\end{array}$ & \\
\hline \multicolumn{8}{|l|}{ Stade clinique (\%) } \\
\hline $\begin{array}{l}\text { cT } \leq 2 \mathrm{a} \\
\text { cT } 2 \mathrm{~b} \\
\text { cT } \geq 2 \mathrm{c}\end{array}$ & $\begin{array}{l}73,0 \\
14,1 \\
12,8\end{array}$ & $\begin{array}{l}76,0 \\
10,8 \\
13,3\end{array}$ & $\begin{array}{l}71,2 \\
15,2 \\
13,6\end{array}$ & $\begin{array}{c}78,5 \\
12,8 \\
8,7\end{array}$ & $\begin{array}{c}68,2 * \\
15,8 \\
16,0 \bullet\end{array}$ & $\begin{array}{l}*=0,02 \\
\bullet=0,02\end{array}$ & \\
\hline \multicolumn{8}{|l|}{ PSA } \\
\hline Moyenne $(\mathrm{ng} / \mathrm{ml}) \pm$ écart type & $9,0 \pm 8,3$ & $8,7 \pm 5,9$ & $8,5 \pm 5,4$ & $9,5 \pm 10,4 *$ & $9,3 \pm 8,8+$ & $\begin{array}{l}*=0,03 \\
\dagger=0,03\end{array}$ & \\
\hline $\begin{array}{l}\mathrm{PSA}<10 \mathrm{ng} / \mathrm{ml}(\%) \\
\mathrm{PSA}=10-20 \mathrm{ng} / \mathrm{ml}(\%) \\
\mathrm{PSA} \geq 20 \mathrm{ng} / \mathrm{ml}(\%)\end{array}$ & $\begin{array}{c}73,9 \\
21,6 \\
4,5 \\
\end{array}$ & $\begin{array}{l}75,6 \\
21,1 \\
3,2 * \\
\end{array}$ & $\begin{array}{c}75,0 \\
20,8 \\
4,1 \\
\end{array}$ & $\begin{array}{c}71,7 \\
23,3 \\
5,1 \\
\end{array}$ & $\begin{array}{c}74,6 \\
20,8 \\
4,6 \\
\end{array}$ & $*<0,001$ & \\
\hline \multicolumn{8}{|l|}{ Classification de D'Amico } \\
\hline $\begin{array}{l}\text { Faible risque (\%) } \\
\text { Risque intermédiaire (\%) } \\
\text { Haut risque (\%) }\end{array}$ & $\begin{array}{c}13,2 \\
80,8 \\
6,0 \\
\end{array}$ & $\begin{array}{c}16,8 \\
79,3 \\
3,9 \\
\end{array}$ & $\begin{array}{c}14,9 \\
80,9 \\
4,2 \\
\end{array}$ & $\begin{array}{c}12,1^{*} \\
83,1 \\
4,8 \\
\end{array}$ & $\begin{array}{c}11,3 \bullet \\
79,1 \\
9,6+ \\
\end{array}$ & $\begin{array}{l}*<0,001 \\
\bullet<0,001 \\
+<0,001\end{array}$ & $\begin{array}{l}У(p<0,001) \\
\nearrow(p<0,001)\end{array}$ \\
\hline \multicolumn{8}{|l|}{ Pièces opératoires } \\
\hline $\begin{array}{l}\text { Score de Gleason }(\%) \text { : } \\
6(3+3)-\text { ISUP } 1 \\
7(3+4)-\text { ISUP } 2 \\
7(4+3)-\text { ISUP } 3 \\
\geq 8(4+4)-\text { ISUP } \geq 4\end{array}$ & $\begin{array}{c}12,7 \\
52,6 \\
26,9 \\
8,1 \\
\end{array}$ & $\begin{array}{c}26,9 \\
40,1 \\
26,5 \\
6,5 \\
\end{array}$ & $\begin{array}{c}21,0 \\
50,0 \\
22,8 \\
6,1 \\
\end{array}$ & $\begin{array}{c}9,2 * \\
52,5 \\
28,3 \\
9,9 \\
\end{array}$ & $\begin{array}{c}3,5+ \\
59,2^{\#} \\
28,8 \\
8,5 \\
\end{array}$ & $\begin{array}{l}*<0,001 \\
+<0,001 \\
\#<0,001\end{array}$ & \\
\hline $\begin{array}{l}\text { Prédominance du grade } 4 \\
\text { (i.e. ISUP } \geq 3 \text { ) (\%) }\end{array}$ & 34,5 & 33,0 & 28,1 & $37,9 *$ & $37,3+$ & $\begin{array}{l}*<0,001 \\
+<0,001\end{array}$ & $\lambda(p=0,01)$ \\
\hline Poids $(\mathrm{g}) \pm$ écart type & $48 \pm 22$ & $47 \pm 24$ & $48 \pm 22$ & $49 \pm 23$ & $46 \pm 20$ & & \\
\hline \multicolumn{8}{|l|}{ Curage ganglionnaire (\%) } \\
\hline $\begin{array}{l}\text { Réalisation en fonction du } \\
\text { groupe de risque de D’Amico: } \\
\text { Faible risque } \\
\text { Risque intermédiaire } \\
\text { Haut risque }\end{array}$ & $\begin{array}{l}14,0 \\
71,6 \\
96,3 \\
\end{array}$ & $\begin{array}{l}14,8 \\
71,5 \\
96,1 \\
\end{array}$ & $\begin{array}{l}13,8 \\
70,9 \\
96,3 \\
\end{array}$ & $\begin{array}{l}16,0 \\
73,9 \\
95,7 \\
\end{array}$ & $\begin{array}{l}12,0 \\
69,9 \\
97,0 \\
\end{array}$ & & \\
\hline $\begin{array}{l}\text { Adéquation de la réalisation du } \\
\text { curage ganglionnaire selon les } \\
\text { recommandations en vigueur: } \\
\text { Faible risque } \\
\text { Risque intermédiaire } \\
\text { Haut risque } \\
\end{array}$ & $\begin{array}{l}83,3 \\
41,8 \\
96,3 \\
\end{array}$ & $\begin{array}{l}77,1 \\
39,9 \\
96,1 \\
\end{array}$ & $\begin{array}{l}79,4 \\
41,0 \\
96,3 \\
\end{array}$ & $\begin{array}{l}88,0 \\
44,0 \\
95,7\end{array}$ & $\begin{array}{l}84,0 \\
41,1 \\
97,0 \\
\end{array}$ & & \\
\hline \multicolumn{8}{|l|}{ Stade pathologiques (\%) } \\
\hline $\begin{array}{l}\text { pT } \leq 2 a \\
\text { pT } 2 b \\
\text { pT } \geq 2 c\end{array}$ & $\begin{array}{c}8,8 \\
5,1 \\
86,1\end{array}$ & $\begin{array}{c}9,3 \\
8,6 \\
82,1\end{array}$ & $\begin{array}{c}14,5 \\
6,3 \\
79,2\end{array}$ & $\begin{array}{c}7,3 \\
1,6^{\circ} \\
91,1\end{array}$ & $\begin{array}{c}5,2^{*} \\
6,1 \\
88,7^{\#}\end{array}$ & $\begin{array}{l}*<0,001 \\
+<0,001 \\
{ }^{\circ}<0,001 \\
\#<0,001\end{array}$ & $\begin{array}{l}\searrow(p=0,004) \\
\nearrow(p=0,003)\end{array}$ \\
\hline Marge positive (\%) & 30,6 & 20,1 & 26,6 & $33,4^{*}$ & $35,2 \bullet$ & $\begin{array}{l}*=0,001 \\
\bullet<0,001\end{array}$ & $\lambda(p<0,001)$ \\
\hline
\end{tabular}

Les $*, \bullet,+,{ }^{\circ}$, \# et $\Omega$ identifient les valeurs significativement différentes des autres valeurs rapportées pour le même item définie en tête de ligne. Les valeurs de $p$ du test utilisé sont présentées en regard.

La tendance monotonique sur les 4 périodes étudiées est présentée comme haussière $(\boldsymbol{\lambda})$ ou baissière $(\boldsymbol{V})$ et la valeur de $p$ du test de Mann-Kendall 